D.O.I: $10.3895 / \mathrm{S} 1808-04482013000200009$

\title{
CRISE FINANCEIRA DE 2008 EM MEIO À GESTÃO DO CAPITAL DE GIRO DE EMPRESAS DO SETOR INDUSTRIAL BRASILEIRO
}

\section{FINANCIAL CRISIS OF 2008 AMID THE MANAGEMENT OF WORKING CAPITAL OF COMPANIES IN THE BRAZILIAN INDUSTRIAL SECTOR}

\author{
Cristiano do Nascimento ${ }^{1}$; Márcia Maria dos Santos Bortolocci Espejo ${ }^{2}$; Simone Bernardes Voese ${ }^{3}$; \\ Elisete Dahmer Pfitscher ${ }^{4}$; João Teles \\ ${ }^{1}$ Universidade Federal do Paraná - UFPR - Curitiba - Brasil \\ cristiano.do.nascimento@live.com \\ ${ }^{2}$ Universidade Federal do Paraná - UFPR - Curitiba - Brasil \\ marciabortolocci@ufpr.br \\ ${ }^{3}$ Universidade Federal do Paraná - UFPR - Curitiba - Brasil \\ sbs1611@ hotmail.com \\ ${ }^{4}$ Universidade Federal de Santa Catarina - UFSC - Florianópolis - Brasil \\ elisete@cse.ufsc.br \\ ${ }^{5}$ Universidade Federal de Santa Catarina - UFSC - Florianópolis - Brasil \\ joaoteles.contab@gmail.com
}

\begin{abstract}
Resumo
Este estudo tem como objetivo principal identificar o nível de liquidez de empresas do setor industrial brasileiro, mediante análise tradicional e dinâmica do capital de giro, em meio ao evento da crise financeira de 2008. A amostra é composta por 31 empresas listadas na Revista Exame Melhores e Maiores de 2009 com ações negociadas na BM\&FBOVESPA (Bolsa de Valores, Mercadorias e Futuros). Executou-se análise descritiva, com abordagem qualitativa e quantitativa. Dentre os resultados, destacam-se: as medidas de dispersão verificadas pela aplicação da estatística descritiva nos indicadores de liquidez tradicionais indicam tendência de redução no distanciamento entre as empresas quanto ao nível de liquidez financeira; mesmo com indicadores positivos, constata-se variação bruta negativa no período VB3 para os indicadores liquidez seca $(L S)$, liquidez corrente ( $L C)$ e liquidez geral ( $L G)$ no nível de 77,4\%; 74,2\%; e 61,3\%, respectivamente; o indicador saldo de tesouraria (ST) demonstra que não ocorreu uma recuperação da liquidez de forma imediata ao final do período da crise, logo a crise durou mais que dois trimestres para o setor industrial brasileiro. A modelagem Fleuriet permitiu visualizar a redução no número de empresas no Tipo 2 de situação financeira sólida, e aumento no Tipo 3 de situação financeira insatisfatória, em períodos relacionados ao advento da crise no Brasil.
\end{abstract}

Palavras-chave: crise financeira; setor industrial brasileiro; capital de giro. 


\section{Introdução}

Uma crise financeira em escala mundial tende a causar, de forma implícita, mudanças nas práticas de gestão e incentivos na busca por alternativas de gestão de processos que favoreçam a geração de resultados satisfatórios, e assim, novos rumos são pensados para que se possa manter e fazer crescer o volume de negócios gerados pela organização. Conforme USA (2011) a crise financeira internacional fora deflagrada em setembro de 2007, prejudicando o desempenho de empresas norte americanas. No Brasil o ano de 2008 apresentou crescimento nos primeiros três trimestres com o PIB em aquecimento progressivo, com ênfase no terceiro trimestre cujo crescimento alcançou $6,8 \%$ sobre o mesmo período de 2007 , sendo que ao final de setembro, a crise financeira internacional passou a afetar a economia brasileira, sobretudo empresas com foco de atuação no mercado internacional (BRASIL, 2010).

O final de 2008 e início de 2009 tende a ter constituído um período de retração no setor industrial brasileiro. Ressalta-se que na variação entre os anos de 2008 e 2009 ocorreu diminuição da produtividade, redução no valor adicionado aos preços básicos, aumento inexpressivo de 0,3902\% no número de empregos formais na indústria de transformação, quando comparado com a variação de 2007 para 2008 em termos de 2,8133\%, entre outros aspectos (BRASIL, 2011).

Neste contexto, desponta a área de finanças e seu poder de influenciar a vida das pessoas e organizações, a qual possibilita a busca por informações na tentativa de se compreender os eventos numa escala macro e micro financeira. No âmbito macro a área de finanças encontra-se intrinsecamente ligada a macroeconomia, cujas questões envolvem fluxos internacionais de capital, sistema financeiro nacional e mercados financeiros; quanto ao âmbito micro, este envolve as finanças das organizações, as quais podem ser divididas em curto prazo e longo prazo (MATIAS, 2007).

A premissa básica deste estudo delineia aspectos investigativos sobre a estrutura financeira de empresas do setor industrial brasileiro. Diante do exposto emerge a seguinte questão de pesquisa: como se encontram o nível de liquidez das empresas atuantes no setor industrial brasileiro em meio ao evento da crise financeira de 2008? Disto isto, configura como objetivo principal identificar o nível de liquidez de empresas do setor industrial brasileiro, mediante análise tradicional e dinâmica do capital de giro, em meio ao evento da crise financeira de 2008.

A crise financeira internacional deflagrada no final de 2008 no Brasil e que afetou de forma incisiva as economias e as perspectivas de crescimento numa escala global, torna as considerações sobre estrutura financeira um tema de significativa importância, pois envolve elementos decisivos em termos de solvência e gestão financeira. Dessa forma, a proposição do presente estudo justificase pela relevância do propósito investigativo acerca do tema estrutura financeira, o qual, quando 
denota perfil de insolvência tende repercutir em desdobramentos sociais, condição que em última instância pode resultar no desemprego, e a partir deste, podem originar-se outros problemas de ordem social.

O presente artigo está estruturado em cinco seções, além desta: referencial teórico composto de indicações literárias no tocante à gestão do capital de giro e crise financeira, metodologia que subsume tipologia da pesquisa, amostras, coleta de dados e procedimentos estatísticos aplicados, resultados do estudo, considerações finais, encerramento com as referências consultadas.

\section{Referencial teórico}

Nesta seção fundamentam-se os constructos análise do capital de giro nas perspectivas dos indicadores tradicionais e dinâmicos e crise financeira, em termos de revisão de literatura, cujas contribuições científicas e conceitos estudados serão utilizados como suporte conceitual para a solução do problema enunciado no presente estudo.

\subsection{Gestão do capital de giro}

A administração do capital de giro, segundo Sousa, Luporini e Souza (1996), consiste num processo contínuo de tomadas de decisões direcionadas, sobretudo, para a preservação da liquidez da empresa que também afeta a rentabilidade. Conforme Assaf Neto e Silva (2010), em meio aos processos de tomada de decisão identificam-se aspectos relacionados às políticas de administração do capital de giro, caracterizadas como agressiva, normal e conservadora, cuja diferença entre tais políticas encontra-se na magnitude do investimento em CDG.

Quanto maior o investimento em CDG, menor o risco de faltas, consequentemente, menor o risco operacional. Contudo, deve-se considerar a existência de um custo na manutenção de CDG, e quanto maior o investimento em CDG, maior o custo de manutenção (ASSAF NETO; SILVA, 2010). As decisões sobre investimentos dependem das condições de financiamento, por conseguinte, decidir sobre a forma de financiamento, sobretudo, volta-se para a perspectiva de maximização da riqueza da empresa. Para poder operar em um período de crise é, conforme Cagliolo, Ferraro e Metilli (2009), indispensável que a empresa tenha liquidez, pois não existe outro mecanismo nem alternativa para operar no curto prazo. Isto garantirá a cobertura da estrutura básica dos custos e gastos, o cumprimento das obrigações financeiras e sociais. Os referidos autores consideram fundamental o saneamento dos passivos, o que só é possível quando se tem liquidez, cuja condição permitirá minimizar o custo de capital.

A magnitude do investimento em ativo circulante constitui política financeira de curto prazo praticada pelas empresas. Ross, Westerfield e Jaffe (2009) relatam que esta política financeira de investimento representa essencialmente o caixa; títulos financeiros; estoques; e volume de contas a 
receber. A empresa pode adotar uma política flexível, realizando investimentos nesses itens mantendo saldos de caixa e geração de estoques além dos níveis considerados necessários, ou até mesmo ampliar o crédito aos seus clientes. De forma contrária, pode adotar uma política restritiva, com foco na manutenção dos níveis mínimos de capital de giro imprescindíveis à sua operação, ou procurando reduzi-los (ROSS; WESTERFIELD; JAFFE, 2009).

Vieira (2008) relata que as empresas dispõem de três fontes principais de recursos para o financiamento das aplicações nos itens do ativo circulante: a primeira delas é formada pelos recursos originários dos seus fornecedores e é decorrente, em grande medida, da própria realização do processo de compras (são consideradas fontes espontâneas de recursos); a segunda fonte é constituída por empréstimos e financiamentos de curto prazo obtidos junto às instituições financeiras; e a terceira fonte é formada pelos empréstimos e financiamentos de longo prazo e pelos recursos do capital próprio. De forma complementar, salienta-se que "uma empresa deve investir em capital de giro enquanto o retorno marginal dos ativos correntes se mantiver acima do custo dos recursos alocados para seu financiamento" (ASSAF NETO; SILVA, 2010, p. 13).

De acordo com Assaf Neto e Silva (2010) quanto maior a participação de recursos de longo prazo e, consequentemente, de CDG, menos arriscada se apresenta a política de capital de giro da empresa. Como asseveram os autores, numa situação de equilíbrio financeiro os recursos de longo prazo devem cobrir o CDG permanente, deixando para o passivo circulante o financiamento das necessidades sazonais dos ativos de curto prazo.

A análise do capital de giro pode ser executada a partir de dois modelos encontrados em livros e estudos empíricos na área de ciências sociais aplicadas, a saber: o modelo tradicional e o modelo dinâmico. No modelo tradicional de gestão do capital de giro, as contas do Balanço Patrimonial são agrupadas mediante adoção de dois critérios, o vertical e o horizontal. A classificação vertical diferencia as contas em relação a sua natureza patrimonial, ou seja, ativo e passivo, quanto à classificação horizontal, esta é temporal, pois as contas são dispostas em conformidade com a ordem decrescente de disponibilidade (ativas) e exigibilidade (passivas) (ASSAF NETO, 2010).

O modelo dinâmico de gestão do capital de giro propõe uma nova classificação horizontal com foco no ciclo de produção, no ciclo econômico e no ciclo financeiro das empresas. O modelo dinâmico utiliza-se da combinação de três variáveis para formar seis modelos de classificação, nos quais se constata o desempenho financeiro e operacional das empresas, a saber: necessidade de capital de giro (NCG), capital de giro (CDG) e saldo de tesouraria (ST). A reclassificação das contas do ativo e passivo é imprescindível para a aplicação do modelo Fleuriet, cujas contas serão “consideradas em relação à realidade dinâmica das empresas, em que as contas são classificadas de 
acordo com o seu ciclo, ou seja, o tempo que leva para se realizar uma rotação" (FLEURIET; KEHDY; BLANC, 2003).

Os estudos de Braga (1991), Marques e Braga (1995), Braga, Nossa e Marques (2004) evidenciam apontamentos sobre os diferentes tipos de Balanços Patrimoniais Gerenciais (BPG) pelos quais se pode visualizar e analisar a liquidez e consequente prática adotada em relação à gestão do capital de giro. O Quadro 1 apresenta uma síntese dos tipos de BPG evidenciando as diferentes estruturas financeiras organizacionais mediante verificação dos valores evidenciados no ativo circulante operacional (ACO), passivo circulante operacional (PCO), ativo permanente (AP), passivo permanente (PP), ativo circulante financeiro (ACF) e passivo circulante financeiro (PCF).

\begin{tabular}{|c|c|c|c|c|}
\multicolumn{5}{c}{ Quadro $1-$ Resumo dos tipos de estrutura financeira } \\
\hline Modelagem & NCG & CDG & ST & RESUMO \\
\hline Tipo 1 & ACO $<$ PCO & PP $>$ AP & ACF $>$ PCF & NCG $<0 ;$ CDG $>0 ;$ ST $>0$ \\
\hline Tipo 2 & ACO $>$ PCO & PP $>$ AP & ACF $>$ PCF & NCG $>0 ;$ CDG $>0 ;$ ST $>0$ \\
\hline Tipo 3 & ACO $>$ PCO & PP $>$ AP & ACF $<$ PCF & NCG $>0 ;$ CDG $>0 ;$ ST $<0$ \\
\hline Tipo 4 & ACO $>$ PCO & PP $<$ AP & ACF $<$ PCF & NCG $>0 ;$ CDG $<0 ;$ ST $<0$ \\
\hline Tipo 5 & ACO $<$ PCO & PP $<$ AP & ACF $<$ PCF & NCG $<0 ;$ CDG $<0 ;$ ST $<0$ \\
\hline Tipo 6 & ACO $<$ PCO & PP $<$ AP & ACF $>$ PCF & NCG $<0 ;$ CDG $<0 ;$ ST $>0$ \\
\hline
\end{tabular}

Fonte: Adaptado de Braga (1991); Marques e Braga (1995); Braga, Nossa e Marques (2004)

O BPG do Tipo 1 apresenta Passivo Permanente maior que o Ativo Permanente, cujo excedente é aplicado no Ativo Circulante, o que denota excelente perfil de liquidez (BRAGA, 1991). O Tipo de 2 de estrutura financeira denota situação financeira sólida, pois todas as variáveis dinâmicas são positivas (BRAGA; NOSSA; MARQUES, 2004). De acordo com Braga (1991), Marques e Braga (1995) o Tipo 3 de BPG indica situação financeira insatisfatória, pois o montante em CDG é inferior a NCG. A tipologia 4 denota que fontes de curto prazo financiam investimentos de longo prazo, tal situação financeira é classificada como ruim por Braga (1991) e péssima para Marques e Braga (1995). No Tipo 5 as variáveis dinâmicas ST; NCG e CDG são negativas, condição que denota situação financeira muito ruim (BRAGA, 1991). A tipologia 6 revela o desvio de sobras do curto prazo para o Ativo Permanente mantendo o ST positivo, embora a NCG e o CDG sejam negativos com NCG menor que o CDG, segundo Braga, Nossa e Marques (2004) tal situação é denominada como arriscada.

\subsection{Efeitos da crise financeira de 2008}

A crise financeira deflagrada no ano de 2007 desponta como a pior crise financeira enfrentada pela economia dos Estados Unidos (EUA), resultando na perda de 3,6 milhões de empregos em 2008 e anúncio da perda de 4,7 milhões de empregos em dezembro de 2009 (USA; 2011). A inadimplência referente a dívidas hipotecárias, tidas como ativos de alto risco, combinadas com o aumento na taxa de juros, desvalorização dos imóveis e desregulamentação financeira, contribuíram para o advento da crise (BEZEMER, 2010). O Congresso dos Estados Unidos criaram 
a Comissão de Inquérito da Crise Financeira - CICF constituída por democratas e republicanos, a qual objetivou investigar e expor à compreensão pública as políticas e práticas que corroboraram para a ocorrência da crise financeira.

O relatório final da CICF, mediante análise técnica dos resultados, expõe as conclusões sobre a ocorrência da crise financeira, a saber: a crise financeira poderia ter sido evitada; falhas na regulação e supervisão financeira mostraram-se devastadoras para a estabilidade financeira dos EUA; falhas nas práticas de governança corporativa e gestão de riscos em instituições financeiras importantes; a combinação de endividamento excessivo, investimentos de risco e a falta de transparência colocou o sistema financeiro do EUA em rota de colisão com a crise; o governo estava mal preparado para enfrentar a crise, dispondo de respostas inconsistentes somadas à incerteza e pânico nos mercados financeiros; ruptura sistemática na responsabilidade e ética; e falhas das agências de notação de crédito, as quais foram catalisadoras da crise pelo fato dos títulos subprime não poderem ser comercializados e vendidos sem um selo de aprovação (USA, 2011).

O ambiente financeiro compõe-se por bancos que atuam nos setores de seguros e imobiliário, os quais destinam-se a gerir todos os tipos de riqueza não-bancárias (fundos de pensão, seguradoras, administradores de fundos, bancos comerciais, imobiliárias, etc), bem como a aceitação de depósitos que geram fluxos de crédito, neste contexto, tais entidades devem ficar, conceitualmente, separadas do setor real, que inclui a administração, empresas e famílias (BEZEMER, 2010). Em meio ao cenário financeiro dos EUA, conforme Cintra e Farhi (2008), a crise revelou a obsolescência das estruturas de supervisão descentralizadas, em razão do nível de imbricação entre as instituições financeiras (bancos, fundos de pensão, seguradoras, fundos de investimento) e mercados (de crédito, de capitais e de derivativos).

Em meio ao cenário nacional a crise financeira de 2008 desencadeou um processo de constrição das condições financeiras em dólares e em reais, cujos efeitos prejudiciais sobre a confiança e a atividade econômica, sobretudo pela depreciação do real, seguido do sintoma da escassez global de liquidez em dólares e da piora dos termos de troca decorrente da queda dos preços de commodities (MESQUITA; TORÓS, 2010). Martins (2010) analisa a elevação no custo do financiamento bancário durante o período da crise financeira de 2008 e constata o aumento no custo do financiamento bancário em meio às restrições impostas por ocasião da contratação do crédito pelas empresas no período da crise.

Conforme Ázara, Passanha e Ázara (2010), o custo do crédito começou a aumentar, por causa da dificuldade de captação externa, pois anteriormente os bancos captavam recursos no exterior a custo menor, mas diante do agravamento da crise nos EUA passam a recorrer aos recursos no mercado interno. Neste contexto, como atestam os autores supracitados, o Banco Central do Brasil adotou medidas no sentido de tentar aumentar a liquidez, como redução de 
depósitos compulsórios (dinheiro que os bancos são obrigados a deixar depositados no Banco Central) no total de $\mathrm{R} \$ 99,8$ bilhões durante a crise; estímulos para que grandes bancos adquirissem carteiras de médios e pequenos; uso das reservas internacionais em linhas de crédito; autorização de comprar, para bancos oficiais, de ações de instituições financeiras privadas sem licitações; venda de dólares das reservas internacionais.

Zani, Zanini e Zani (2010) destacam que as empresas exportadoras brasileiras que apostaram na valorização da moeda brasileira se depararam com significativo impacto em seus resultados e exemplifica ao citar a Aracruz, Votorantim, Sadia e Embraer. Sena et al (2009) investigaram como as empresas lidam com a crise e dentre os resultados destacam não existir diferença significativa com relação à classificação entre otimistas e neutros, se comparados ao período pré-crise e após seu início, podendo indicar uma tendência a esconder os acontecimentos ruins ou uma esperança de melhora no futuro.

Conforme Bizotto et al (2011) a crise influenciou os resultados por meio da redução do faturamento e redução dos preços, os quais configuram como sendo os principais problemas enfrentados. Os referidos autores salientam que a empresa do ramo alimentício analisada, em razão de fornecer produtos de necessidade básica, mostrou-se menos susceptível aos impactos de ordem financeira decorrentes da crise comparativamente a empresas de outros segmentos.

\section{Metodologia}

Este estudo fundamenta-se no propósito investigativo de aspectos que relacionam a gestão do capital de giro, o cenário da crise financeira de 2008 e as empresas do setor industrial com ações negociadas em bolsa e concomitantemente listadas entre as 500 maiores empresas brasileiras. Tratase de pesquisa com a característica descritiva, pois procura descrever e analisar mudanças ocorridas em meio às práticas de gestão do capital de giro e aquelas ocorridas na estrutura financeira das empresas do setor industrial brasileiro mediante as perspectivas tradicional e dinâmica de análise do capital de giro. Trata-se de estudo ex post facto, no qual o pesquisador não tem controle sobre as variáveis no sentido de poder manipulá-las, pode apenas relatar o que aconteceu ou que está acontecendo (COOPER; SCHINDLER, 2003).

O estudo tem característica longitudinal, a qual segundo Cooper e Schindler (2003), permite acompanhar mudanças ao longo do tempo, a partir da análise por três trimestres anteriores ao período da crise no Brasil, ou seja, de janeiro a setembro de 2008; período de crise deflagrada entre outubro de 2008 até fevereiro de 2009; e três trimestres posteriores a crise, de abril a dezembro de 2009.

A análise de dados é qualitativa e quantitativa. A abordagem qualitativa permite inferir sobre os aspectos inerentes ao evento da crise financeira e a análise aplicada ao conjunto de 
estruturas financeiras identificadas. A abordagem quantitativa permite visualizar, mediante mensuração, a representatividade dos resultados encontrados. A utilização do método quantitativo, para a análise dos dados, "representa, em princípio, a intenção de garantir a precisão dos resultados, evitar distorções de análise e interpretação, possibilitando, consequentemente, uma margem de segurança quanto às inferências" (RICHARDSON, 1999, p. 70). O tratamento estatístico estruturase a partir de estatística descritiva dos dados com base em medidas de dispersão, considerando o valor máximo e mínimo para cada indicador, e então se executa o cálculo da média; variância; e desvio padrão, para posterior análise.

A análise do capital de giro proposta neste estudo compreende as perspectivas tradicional e dinâmica, na primeira são utilizados os indicadores liquidez seca, liquidez corrente e liquidez geral calculados a partir dos Balanços Patrimoniais, na segunda faz-se uso dos indicadores saldo de tesouraria; necessidade de capital de giro e capital de giro calculados a partir da reclassificação das contas dos Balanços Patrimoniais Gerenciais (BPG), em conformidade com os pressupostos do modelo Fleuriet. O recurso técnico utilizado para o cálculo dos indicadores foi o software Microsoft Office Excel 2007. No intuito de verificar o comportamento dos indicadores tradicionais e dinâmicos calcula-se a variação bruta (VB) entre os trimestres, cujas siglas correspondentes aos períodos calculados encontram-se dispostas no Quadro 2, a seguir:

Quadro 2 - Siglas que representam a variação bruta entre os trimestres

\begin{tabular}{|c|c|}
\hline Variação Bruta - VB & Período correspondente \\
\hline VB1 & $2^{\mathbf{o}}$ trimestre de 2008 menos $1^{\circ}$ trimestre de 2008 \\
\hline VB2 & $3^{\circ}$ trimestre de 2008 menos $2^{\circ}$ trimestre de 2008 \\
\hline VB3 & $4^{\mathbf{o}}$ trimestre de 2008 menos $3^{\circ}$ trimestre de 2008 \\
\hline VB4 & $1^{\mathbf{o}}$ trimestre de 2009 menos $4^{\circ}$ trimestre de 2008 \\
\hline VB5 & $2^{\mathbf{o}}$ trimestre de 2009 menos $1^{\circ}$ trimestre de 2009 \\
\hline VB6 & $3^{\circ}$ trimestre de 2009 menos $2^{\circ}$ trimestre de 2009 \\
\hline VB7 & $4^{\circ}$ trimestre de 2009 menos $3^{\circ}$ trimestre de 2009 \\
\hline
\end{tabular}

Fonte: Feito pelos autores (2010)

A amostra selecionada para este estudo compreende as empresas do setor industrial brasileiro presentes na lista das 500 maiores empresas em vendas atuantes no Brasil, com ações negociadas na BM\&FBOVESPA, e com base informacional completa para fins de execução deste estudo, resultando num conjunto de 31 empresas. O Quadro 3 expõe as empresas componentes da amostra, pelo nome de Pregão, classificadas por ramo de atividade conforme periódico Exame (2009), a seguir: 
Quadro 3 - Amostra da pesquisa

\begin{tabular}{|c|c|c|c|}
\hline & Bens de Consumo & GRENDENE & Têxteis \\
\hline BRF FOODS & Bens de Consumo & GUARARAPES & Têxteis \\
\hline JMBEV & Bens de Consumo & ALPARGATAS & Têxteis \\
\hline M.DIASBRANCO & Bens de Consumo & VICUNHA TEXT & Têxteis \\
\hline MINERVA & Bens de Consumo & CIA HERING & Têxteis \\
\hline NATURA & Bens de Consumo & EMBRAER & Autoindústria \\
\hline SOUZA CRUZ & Bens de Consumo & IOCHP-MAXION & Autoindústria \\
\hline ACOS VILL & Siderurgia e Metalurgia & MARCOPOLO & Autoindústria \\
\hline SID NACIONAL & Siderurgia e Metalurgia & RANDON PART & Autoindústria \\
\hline GERDAU & Siderurgia e Metalurgia & ITAUTEC & Indústria Digital \\
\hline TUPY & Siderurgia e Metalurgia & TOTVS & Indústria Digital \\
\hline USIMINAS & Siderurgia e Metalurgia & UOL & Indústria Digital \\
\hline DURATEX & Indústria da Construção & FIBRIA & Papel e Celulose \\
\hline GAFISA & Indústria da Construção & KLABIN S/A & Papel e Celulose \\
\hline MRV & Indústria da Construção & SUZANO PAPEL & Papel e Celulose \\
\hline BRASKEM & Química e petroquímica & & \\
\hline
\end{tabular}

Fonte: Exame (2009)

O levantamento dos dados da amostra foi obtido na base da Economática® Software para Investimentos Ltda. O período de aplicação deste estudo compreende os quatro trimestres de 2008 e 2009.

\section{Resultados do estudo}

Nesta seção apresenta-se o estudo realizado sobre estrutura financeira das empresas do setor industrial brasileiro com foco de análise no período da crise financeira de 2008. O primeiro tópico expõe aspectos relacionados aos níveis de liquidez na perspectiva tradicional de análise do capital de giro, o segundo tópico investiga os níveis de liquidez na perspectiva da modelagem Fleuriet, seguido de uma análise fundamentada na tipologia de Fleuriet.

\subsection{Indicadores tradicionais do capital de giro}

A proposição da análise tradicional do capital de giro estrutura-se a partir dos resultados obtidos pelos indicadores financeiros de liquidez, a saber: liquidez seca (LS), liquidez corrente (LC) e liquidez geral (LG). Cujos indicadores têm a capacidade de contribuir com informações úteis em meio ao processo de tomada de decisão no tocante aos aspectos relacionados à gestão do capital de giro. Calculam-se os indicadores supracitados nos oito trimestres de cada empresa da amostra, ou seja, analisa-se 248 Balanços Patrimoniais. Na Tabela 1 apresenta-se a quantidade e o percentual referente aos quocientes dos indicadores de liquidez analisados diferenciando-os entre indicador menor e maior que 1. 
Tabela 1 - Frequência nos níveis de liquidez dos indicadores financeiros tradicionais por trimestre $(\mathrm{Tr})$

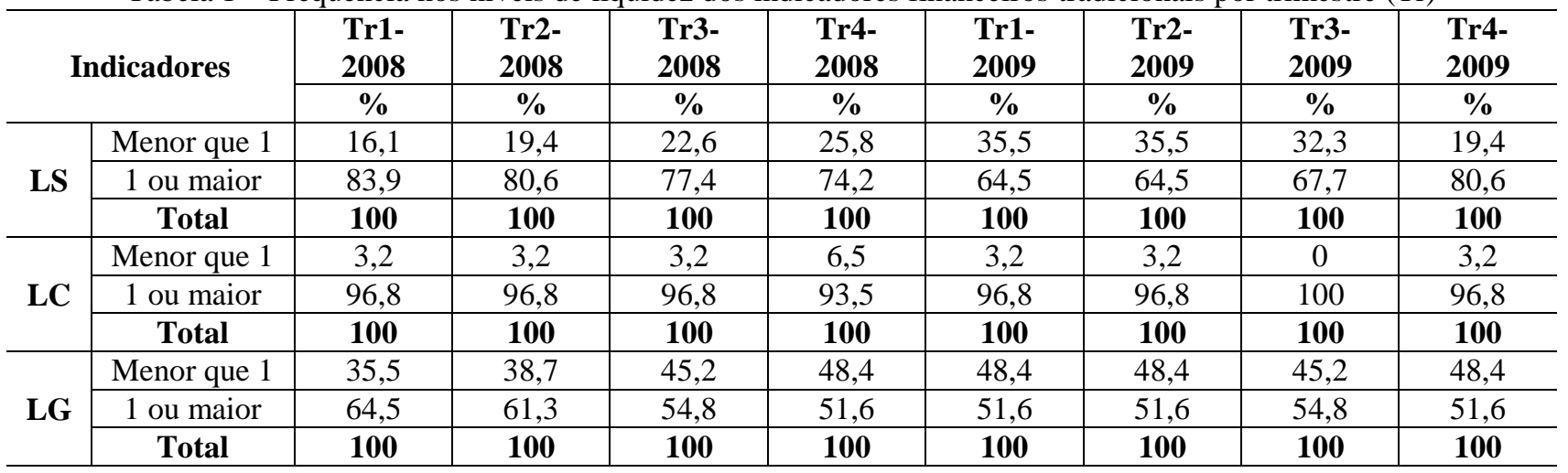

$* \%=$ percentual

Fonte: Dados da pesquisa (2010)

Conforme Tabela 1, o número de indústrias com quociente menor que 1 aumentou ao longo de 2008 até o final do primeiro semestre de 2009, para o indicador LS, com representatividade de $16,1 \%$ no primeiro trimestre de 2008 e alcançando 35,5\% no segundo trimestre de 2009. Da mesma forma, constata-se o crescente número de empresas com o indicador LG menor que 1 ao longo de 2008 até o final do primeiro semestre de 2009, alcançando representativos 48,4\% da amostra. Logo, infere-se que os indicadores LS e LG denotam a possibilidade da falta de recursos disponíveis ao atendimento de compromissos assumidos para o curto prazo em meio ao período cuja crise financeira fora deflagrada no ambiente macroeconômico nacional. Na Tabela 2 expõem-se os dados da estatística descritiva aplicada aos indicadores contábeis financeiros tradicionais.

Tabela 2 - Estatística descritiva referente à liquidez das empresas da amostra

\begin{tabular}{|c|c|c|c|c|c|c|c|}
\hline Indicador & Trimestres & $\mathbf{N}^{*}$ & Mínimo & Máximo & Média & Variância & Desvio Padrão \\
\hline \multirow{8}{*}{ 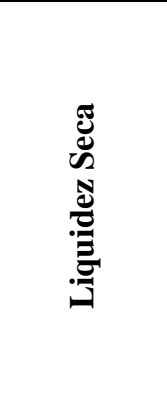 } & $\operatorname{Tr} 1-2008$ & 31 & 0,69284511 & 6,20752213 & 1,87826314 & 1,71606856 & 1,309988 \\
\hline & Tr2-2008 & 31 & 0,56036693 & 8,75298583 & 1,82892556 & 2,50242606 & 1,58190583 \\
\hline & Tr3-2008 & 31 & 0,58471697 & 6,83584311 & 1,67799034 & 1,50741331 & 1,22776761 \\
\hline & Tr4-2008 & 31 & 0,58615541 & 5,12386884 & 1,52757906 & 0,99331911 & 0,99665396 \\
\hline & Tr1-2009 & 31 & 0,6725108 & 5,66176763 & 1,50256522 & 1,13550197 & 1,06559935 \\
\hline & Tr2-2009 & 31 & 0,7186789 & 4,77127117 & 1,55864969 & 0,99863612 & 0,99931783 \\
\hline & Tr3-2009 & 31 & 0,750332 & 4,5896972 & 1,6783452 & 0,90323756 & 0,95038811 \\
\hline & Tr4-2009 & 31 & 0,7034213 & 5,43100773 & 1,62669893 & 0,85002731 & 0,92196926 \\
\hline \multirow{8}{*}{ 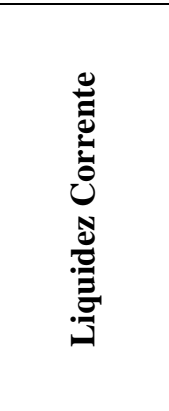 } & Tr1-2008 & 31 & 0,90244962 & 6,79397147 & 2,49089549 & 1,66380675 & 1,28988633 \\
\hline & Tr2-2008 & 31 & 0,81111827 & 9,84054717 & 2,47147489 & 2,66714506 & 1,63313963 \\
\hline & Tr3-2008 & 31 & 0,7585898 & 7,72202074 & 2,29727497 & 1,6569856 & 1,28723953 \\
\hline & Tr4-2008 & 31 & 0,78199176 & 5,67551259 & 2,10323276 & 1,08060913 & 1,03952351 \\
\hline & Tr1-2009 & 31 & 0,93427236 & 6,14189751 & 2,06435364 & 1,17475108 & 1,08385935 \\
\hline & Tr2-2009 & 31 & 0,97344927 & 5,22699596 & 2,11081103 & 1,12829049 & 1,06221019 \\
\hline & Tr3-2009 & 31 & 1,0364754 & 4,8274264 & 2,20283023 & 0,93238962 & 0,96560324 \\
\hline & Tr4-2009 & 31 & 0,9666623 & 6,00738261 & 2,12504783 & 0,90668593 & 0,95220057 \\
\hline \multirow{5}{*}{ 矛 } & Tr1-2008 & 31 & 0,54001321 & 4,920825 & 1,49441915 & 1,34880823 & 1,16138204 \\
\hline & Tr2-2008 & 31 & 0,57770604 & 5,34031468 & 1,47651741 & 1,3775151 & 1,17367589 \\
\hline & Tr3-2008 & 31 & 0,57077174 & 4,2462339 & 1,29782829 & 0,81314586 & 0,90174601 \\
\hline & Tr4-2008 & 31 & 0,36769546 & 3,97360267 & 1,22210688 & 0,68638599 & 0,82848415 \\
\hline & Tr1-2009 & 31 & 0,36598333 & 4,63509194 & 1,24003011 & 0,78655403 & 0,88687881 \\
\hline
\end{tabular}




\begin{tabular}{c|c|c|c|c|c|c|c}
\hline Indicador & Trimestres & N* & Mínimo & Máximo & Média & Variância & Desvio Padrão \\
\hline \multirow{2}{*}{} & Tr2-2009 & 31 & 0,37200573 & 4,18257044 & 1,26226193 & 0,71983487 & 0,84843083 \\
\cline { 2 - 8 } & Tr3-2009 & 31 & 0,44917863 & 4,25608861 & 1,27814572 & 0,68379439 & 0,82691861 \\
\cline { 2 - 8 } & Tr4-2009 & 31 & 0,45131075 & 5,24024341 & 1,2619473 & 0,80402623 & 0,8966751 \\
\hline
\end{tabular}

$\mathrm{N}^{*}:$ amostra

Fonte: Dados da pesquisa (2010)

A Tabela 2 apresenta os dados resultantes da aplicação da estatística descritiva em relação aos indicadores LS e LC, os quais denotam tendência de redução das medidas de dispersão, média; variância e desvio padrão, no terceiro trimestre de 2008, cuja redução acentua-se no período correspondente ao de ocorrência da crise em meio ao ambiente macroeconômico nacional, em que, na média, verifica-se a redução na disponibilidade de recursos para o pagamento de dívidas de curto prazo, comparativamente aos primeiros três trimestres de 2008. Quanto ao indicador LG, salienta-se a diminuição dos valores correspondentes a variância e desvio padrão a partir do quarto trimestre de 2008, condição que remete a inferir acerca da tendência de redução no distanciamento entre as empresas em termos de disponibilidade de recursos para pagamento das dívidas de curto e de longo prazo.

Os resultados obtidos a partir do cálculo dos indicadores tradicionais aplicados aos oito trimestres analisados revelam uma tendência de redução em relação ao nível de liquidez empresarial. Porém, mesmo com um perfil de liquidez positivo, a empresa pode estar sujeita a redução na sua capacidade de honrar os compromissos assumidos, deste modo, calcula-se a variação bruta trimestral no sentido de identificar o montante de indústrias com redução na disponibilidade de recursos, incluindo aquelas que não tiveram indicador menor que 1 em quais quer dos indicadores tradicionais analisados. Dessa forma, apresenta-se o montante de indústrias com redução ou aumento dos recursos disponíveis no contexto da composição de sua estrutura financeira. Na Tabela 3 demonstram-se, em termos quantitativos e percentuais, as indústrias com variação bruta (VB) negativa e positiva em relação aos indicadores tradicionais.

Tabela 3 - Variação bruta por trimestre (Tr) dos indicadores financeiros tradicionais

\begin{tabular}{|c|c|c|c|c|c|c|c|c|c|c|c|c|c|c|c|}
\hline \multirow{2}{*}{\multicolumn{2}{|c|}{ Indicadores }} & \multicolumn{2}{|c|}{ VB1 } & \multicolumn{2}{|c|}{ VB2 } & \multicolumn{2}{|c|}{ VB3 } & \multicolumn{2}{|c|}{ VB4 } & \multicolumn{2}{|c|}{ VB5 } & \multicolumn{2}{|c|}{ VB6 } & \multicolumn{2}{|c|}{ VB7 } \\
\hline & & $\mathrm{Q}^{*}$ & $\%$ & $\mathrm{Q}^{*}$ & $\%$ & $\mathrm{Q}^{*}$ & $\%$ & $\mathrm{Q}^{*}$ & $\%$ & $\mathrm{Q}^{*}$ & $\%$ & $\mathrm{Q}^{*}$ & $\%$ & $\mathrm{Q}^{*}$ & $\%$ \\
\hline \multirow{3}{*}{$\begin{array}{l}\text { Liquidez } \\
\text { Seca }\end{array}$} & $\begin{array}{l}\text { Variação } \\
\text { Negativa }\end{array}$ & 20 & 64,5 & 21 & 67,7 & 24 & 77,4 & 17 & 54,8 & 10 & 32,3 & 9 & 29,0 & 14 & 45,2 \\
\hline & Variação Positiva & 11 & 35,5 & 10 & 32,3 & 7 & 22,6 & 14 & 45,2 & 21 & 67,7 & 22 & 71,0 & 17 & 54,8 \\
\hline & Total & 31 & 100 & 31 & 100 & 31 & 100 & 31 & 100 & 31 & 100 & 31 & 100 & 31 & 100 \\
\hline \multirow{3}{*}{$\begin{array}{l}\text { Liquidez } \\
\text { Corrente }\end{array}$} & $\begin{array}{r}\text { Variação } \\
\text { Negativa } \\
\end{array}$ & 17 & 54,8 & 21 & 67,7 & 23 & 74,2 & 17 & 54,8 & 13 & 41,9 & 9 & 29,0 & 15 & 48,4 \\
\hline & Variação Positiva & 14 & 45,2 & 10 & 32,3 & 8 & 25,8 & 14 & 45,2 & 18 & 58,1 & 22 & 71,0 & 16 & 51,6 \\
\hline & Total & 31 & 100 & 31 & 100 & 31 & 100 & 31 & 100 & 31 & 100 & 31 & 100 & 31 & 100 \\
\hline \multirow{3}{*}{$\begin{array}{c}\text { Liquidez } \\
\text { Geral }\end{array}$} & $\begin{array}{c}\text { Variação } \\
\text { Negativa } \\
\end{array}$ & 17 & 54,8 & 20 & 64,5 & 19 & 61,3 & 13 & 41,9 & 8 & 25,8 & 11 & 35,5 & 17 & 54,8 \\
\hline & Variação Positiva & 14 & 45,2 & 11 & 35,5 & 12 & 38,7 & 18 & 58,1 & 23 & 74,2 & 20 & 64,5 & 14 & 45,2 \\
\hline & Total & 31 & 100 & 31 & 100 & 31 & 100 & 31 & 100 & 31 & 100 & 31 & 100 & 31 & 100 \\
\hline
\end{tabular}


Salienta-se que o período VB3 corresponde à diferença entre o quarto e terceiro trimestres de 2008, no qual, em conformidade com o exposto na Tabela 3, visualiza-se a maior representatividade percentual de empresas com variação bruta negativa para os indicadores LS, LC e LG no nível de 77,4\%; 74,2\%; e 61,3\%, respectivamente. Contudo, ressalta-se o volume significativo de empresas que apresenta redução na disponibilidade de recursos, mediante indicadores LS, LC e LG com 54,8\%; 54,8\%; e 41,9\%, respectivamente, para o período VB4. Dessa forma, constata-se a redução no volume de recursos disponíveis no contexto da composição da estrutura financeira das indústrias no período em que a crise financeira foi deflagrada.

\subsection{Indicadores dinâmicos do capital de giro}

Os 248 Balanços Patrimoniais analisados foram reclassificados no intuito de atender os preceitos da modelagem Fleuriet, dos quais se obteve 248 Balanços Patrimoniais Gerenciais (BPG), para fins de identificação das variáveis dinâmicas ST; NCG; e CDG. Na Tabela 4 apresentam-se os resultados negativos e positivos referentes às variáveis dinâmicas de análise do capital de giro.

Tabela 4 - Frequência de resultados negativos e positivos por variável dinâmica nos trimestres (Tr)

\begin{tabular}{|c|c|c|c|c|c|c|c|c|c|}
\hline \multirow{2}{*}{\multicolumn{2}{|c|}{ Indicadores }} & Tr1-2008 & Tr2-2008 & Tr3-2008 & Tr4-2008 & Tr1-2009 & $\operatorname{Tr2-2009}$ & Tr3-2009 & Tr4-2009 \\
\hline & & $\%$ & $\%$ & $\%$ & $\%$ & $\%$ & $\%$ & $\%$ & $\%$ \\
\hline \multirow{3}{*}{ 证 } & Negativo & 25,8 & 32,3 & 29,0 & 35,5 & 45,2 & 41,9 & 35,5 & 32,3 \\
\hline & Positivo & 74,2 & 67,7 & 71,0 & 64,5 & 54,8 & 58,1 & 64,5 & 67,7 \\
\hline & Total & 100 & 100 & 100 & 100 & 100 & 100 & 100 & 100 \\
\hline \multirow{3}{*}{$\begin{array}{l}\text { U } \\
\mathbf{Z}\end{array}$} & Negativo & 3,2 & 3,2 & 3,2 & 6,5 & 9,7 & 9,7 & 6,5 & 12,9 \\
\hline & Positivo & 96,8 & 96,8 & 96,8 & 93,5 & 90,3 & 90,3 & 93,5 & 87,1 \\
\hline & Total & 100 & 100 & 100 & 100 & 100 & 100 & 100 & 100 \\
\hline \multirow{3}{*}{ 己ٌ } & Negativo & 3,2 & 3,2 & 3,2 & 3,2 & 0 & 3,2 & 0 & 3,2 \\
\hline & Positivo & 96,8 & 96,8 & 96,8 & 96,8 & 100 & 96,8 & 100 & 96,8 \\
\hline & Total & 100 & 100 & 100 & 100 & 100 & 100 & 100 & 100 \\
\hline
\end{tabular}

$\%:$ percentual

Fonte: Dados da pesquisa (2010)

De acordo com a Tabela 4, a variável dinâmica ST apresenta maior número de empresas com saldo negativo no primeiro trimestre de 2009 , alcançando $45,2 \%$ da amostra, cuja redução deste montante mostra-se lenta ao longo de 2009, ou seja, não ocorreu uma recuperação dos níveis adequados do saldo de caixa de forma imediata ao final do período de crise no Brasil. As variáveis dinâmicas NCG e CDG não demonstram diferença significativa na variação do número de empresas com saldo negativo a partir das contas dispostas no BPG. A Tabela 5 apresenta a variação bruta por trimestre aplicada aos indicadores dinâmicos de análise do capital de giro. 
Tabela 5 - Variação bruta por trimestre (Tr) dos indicadores dinâmicos de análise do capital de giro

\begin{tabular}{|c|c|c|c|c|c|c|c|c|c|c|c|c|c|c|c|}
\hline \multirow{2}{*}{\multicolumn{2}{|c|}{ Indicadores }} & \multicolumn{2}{|c|}{ VB1 } & \multicolumn{2}{|c|}{ VB2 } & \multicolumn{2}{|c|}{ VB3 } & \multicolumn{2}{|c|}{ VB4 } & \multicolumn{2}{|c|}{ VB5 } & \multicolumn{2}{|c|}{ VB6 } & \multicolumn{2}{|c|}{ VB7 } \\
\hline & & $\mathbf{Q}^{*}$ & $\%$ & $\mathbf{Q}^{*}$ & $\%$ & $\mathbf{Q}^{*}$ & $\%$ & $\mathbf{Q}^{*}$ & $\%$ & $\mathbf{Q}^{*}$ & $\%$ & $\mathbf{Q}^{*}$ & $\%$ & $\mathbf{Q}^{*}$ & $\%$ \\
\hline \multirow{3}{*}{ 量 } & $\begin{array}{l}\text { Variação } \\
\text { Negativa }\end{array}$ & 13 & 41,9 & 17 & 54,8 & 25 & 80,6 & 18 & 58,1 & 9 & 29,0 & 12 & 38,7 & 17 & 54,8 \\
\hline & $\begin{array}{l}\text { Variação } \\
\text { Positiva }\end{array}$ & 18 & 58,1 & 14 & 45,2 & 6 & 19,4 & 13 & 41,9 & 22 & 71,0 & 19 & 61,3 & 14 & 45,2 \\
\hline & Total & 31 & 100 & 31 & 100 & 31 & 100 & 31 & 100 & 31 & 100 & 31 & 100 & 31 & 100 \\
\hline \multirow{3}{*}{$\begin{array}{l}\vdots \\
\text { Z } \\
\end{array}$} & $\begin{array}{l}\text { Variação } \\
\text { Negativa }\end{array}$ & 14 & 45,2 & 9 & 29,0 & 7 & 22,6 & 19 & 61,3 & 24 & 77,4 & 13 & 41,9 & 19 & 61,3 \\
\hline & $\begin{array}{l}\text { Variação } \\
\text { Positiva }\end{array}$ & 17 & 54,8 & 22 & 71,0 & 24 & 77,4 & 12 & 38,7 & 7 & 22,6 & 18 & 58,1 & 12 & 38,7 \\
\hline & Total & 31 & 100 & 31 & 100 & 31 & 100 & 31 & 100 & 31 & 100 & 31 & 100 & 31 & 100 \\
\hline \multirow{3}{*}{ อి } & $\begin{array}{l}\text { Variação } \\
\text { Negativa }\end{array}$ & 11 & 35,5 & 7 & 22,6 & 18 & 58,1 & 22 & 71,0 & 18 & 58,1 & 7 & 22,6 & 13 & 41,9 \\
\hline & $\begin{array}{l}\text { Variação } \\
\text { Positiva }\end{array}$ & 20 & 64,5 & 24 & 77,4 & 13 & 41,9 & 9 & 29,0 & 13 & 41,9 & 24 & 77,4 & 18 & 58,1 \\
\hline & Total & 31 & 100 & 31 & 100 & 31 & 100 & 31 & 100 & 31 & 100 & 31 & 100 & 31 & 100 \\
\hline
\end{tabular}

$\mathrm{Q}^{*}:$ quantidade

$\%$ : percentual

Fonte: Dados da pesquisa (2010)

O cálculo da variação bruta dos indicadores dinâmicos, exposto de forma resumida na Tabela 5, permite visualizar o montante de empresas que tiveram aumento ou redução na disponibilidade de recursos no contexto da gestão do capital de giro. Destaca-se o aumento no número de empresas componentes da amostra com variação bruta negativa para as variáveis dinâmicas ST e CDG, com a representatividade de $80,6 \%$ e 58,1\%, respectivamente, referente à VB3, cujo período em análise é reconhecido como o de início da crise no Brasil. Salienta-se que os indicadores ST e CDG evidenciam o aumento das dificuldades financeiras entre as empresas analisadas, nas quais se identifica um volume representativo de ocorrências de variação bruta negativa no período VB4.

A variável dinâmica NCG, conforme Tabela 5, evidencia redução no número de empresas com variação negativa com $22,6 \%$ da amostra em VB3, o menor percentual ao longo período analisado. Infere-se que os recursos aplicáveis no ciclo operacional, diante a crise deflagrada, tendem a terem sido destinados para outra forma de aplicação, podendo ser financeira ou permanente, no sentido de ajuste ao fluxo de recursos no contexto da estrutura financeira das empresas. Tal fato remete a considerar a iniciativa em alterar a configuração da estrutura financeira em meio ao período da crise como forma de adequar às finanças organizacionais a possíveis dificuldades na manutenção dos níveis de liquidez.

\subsection{Análise da tipologia Fleuriet}

Na Tabela 6 evidencia-se a representatividade inerente a estrutura financeira das empresas do setor industrial analisadas a partir da tipologia de Fleuriet. 
Tabela 6 - Representatividade da tipologia de Fleuriet ao longo dos oito trimestres

\begin{tabular}{|c|c|c|c|c|c|c|c|c|c|c|c|c|c|c|}
\hline \multirow{3}{*}{$\begin{array}{c}\text { Trimestres } \\
\text { (Tr) }\end{array}$} & \multicolumn{12}{|c|}{ Tipologia de Fleuriet } & \multirow{2}{*}{\multicolumn{2}{|c|}{ Total }} \\
\hline & \multicolumn{2}{|c|}{1} & \multicolumn{2}{|c|}{2} & \multicolumn{2}{|c|}{3} & \multicolumn{2}{|c|}{4} & \multicolumn{2}{|c|}{5} & \multicolumn{2}{|c|}{6} & & \\
\hline & $\mathrm{Q}^{*}$ & $\%$ & $\mathrm{Q}^{*}$ & $\%$ & $\mathrm{Q}^{*}$ & $\%$ & $\mathrm{Q}^{*}$ & $\%$ & $\mathrm{Q}^{*}$ & $\%$ & $\mathrm{Q}^{*}$ & $\%$ & $\mathrm{Q}^{*}$ & $\%$ \\
\hline Tr1-2008 & 1 & 3,2 & 22 & 71,0 & 7 & 22,6 & 1 & 3,2 & 0 & 0 & 0 & 0 & 31 & 100 \\
\hline Tr2-2008 & 1 & 3,2 & 20 & 64,5 & 9 & 29,0 & 1 & 3,2 & 0 & 0 & 0 & 0 & 31 & 100 \\
\hline Tr3-2008 & 1 & 3,2 & 21 & 67,7 & 8 & 25,8 & 1 & 3,2 & 0 & 0 & 0 & 0 & 31 & 100 \\
\hline Tr4-2008 & 2 & 6,5 & 18 & 58,1 & 9 & 29,0 & 2 & 6,5 & 0 & 0 & 0 & 0 & 31 & 100 \\
\hline Tr1-2009 & 2 & 6,5 & 15 & 48,4 & 13 & 41,9 & 0 & 0 & 1 & 3,2 & 0 & 0 & 31 & 100 \\
\hline Tr2-2009 & 2 & 6,5 & 15 & 48,4 & 13 & 41,9 & 0 & 0 & 0 & 0 & 1 & 3,2 & 31 & 100 \\
\hline Tr3-2009 & 2 & 6,5 & 18 & 58,1 & 10 & 32,3 & 0 & 0 & 0 & 0 & 1 & 3,2 & 31 & 100 \\
\hline Tr4-2009 & 3 & 9,7 & 17 & 54,8 & 10 & 32,3 & 0 & 0 & 0 & 0 & 1 & 3,2 & 31 & 100 \\
\hline Total & 14 & 5,6 & 146 & 58.9 & 79 & 31,9 & 5 & 2,0 & 1 & 0,4 & 3 & 1,2 & 248 & 100 \\
\hline
\end{tabular}

$\mathrm{Q}^{*}$ : quantidade

$\%$ : percentual

Fonte: Dados da pesquisa (2010)

Os dados expostos na Tabela 6 permitem identificar que a estrutura financeira do Tipo 2 predomina entre os 248 BPG analisados com 58,9\% da amostra, cuja condição financeira evidencia uma situação financeira sólida, pois dispõem das variáveis ST; NCG; e CDG, com resultado positivo (BRAGA; NOSSA; MARQUES, 2004). O Tipo 1 de BPG tido como excelente por Braga (1991), constitui 5,6\% dos demonstrativos analisados. O perfil Tipo 3 representa 31,9\% dos BPG analisados e indica situação financeira insatisfatória pelo fato do montante em CDG ser inferior a NCG (BRAGA, 1991). As empresas com modelagem Tipo 4 tem representatividade de $2 \%$ dos BPG analisados, segundo Marques e Braga (1995), apresentam uma situação financeira ruim. A modelagem Tipo 5 constitui $0,4 \%$ da amostra de BPG, a qual demonstra uma situação financeira muito ruim (BRAGA, 1991). A tipologia 6, com 1,2\%, dispõe de empresas que mantém saldo positivo em tesouraria a partir do desvio das sobras de recursos de curto prazo para o ativo circulante, cuja situação tende a não se manter ao longo do tempo (BRAGA, 1991).

O perfil Tipo 2 denota tendência de redução no conjunto de empresas a partir do quarto trimestre de 2008, sendo que no primeiro e segundo trimestres de 2009 alcançou 48,4\% da amostra comparativamente aos $67,7 \%$ do terceiro trimestre de 2008, o qual antecedeu o advento da crise financeira no Brasil. As tipologias 4; 5; e 6 denotam reduzida representatividade dentre os demonstrativos analisados. No primeiro trimestre de 2009 desponta o aumento no número de empresas com tipologia 3, tido como estrutura financeira que demanda atenção no sentido de se evitar o aumento da diferença entre o CDG e a NCG.

\section{Considerações finais}

Este estudo foi desenvolvido a partir dos dados dispostos nos Balanços Patrimoniais de 31 empresas atuantes no setor industrial brasileiro. O objetivo principal foi alcançado, mediante análise dos dados organizados em tabelas e quadros. Dessa forma, o foco da pesquisa volta-se para a análise do capital de giro fundamentado nos parâmetros previstos nas perspectivas tradicional e 
dinâmica de análise, a primeira com o perfil estanque de um determinado momento no tempo, e a segunda que permite inferir sobre as condições futuras da empresa, em meio ao contexto da crise financeira de 2008.

Destaca-se que a aplicação da estatística descritiva em relação aos indicadores LS e LC denota tendência de redução das medidas de dispersão, média; variância e desvio padrão, no terceiro trimestre de 2008, ou seja, constatam-se sinais da crise no setor um trimestre antes da crise ser declarada oficialmente no Brasil, cuja redução acentua-se no período correspondente ao de ocorrência da crise em meio ao ambiente macroeconômico nacional, enquanto que o indicador LG apresenta diminuição dos valores na variância e desvio padrão a partir do quarto trimestre de 2008, em que, na média, verifica-se a redução na disponibilidade de recursos, logo a situação financeira das empresas tende a se tornar mais homogenia no tocante ao nível de solvência.

Os resultados evidenciados pelo indicador ST demonstram que não ocorreu uma recuperação dos níveis adequados do saldo de caixa de forma imediata ao final do período de crise no Brasil, logo a crise durou mais que dois trimestres para o setor industrial brasileiro. Contatou-se o aumento no número de empresas com variação bruta negativa para as variáveis dinâmicas ST e CDG, com a representatividade de $80,6 \%$ e $58,1 \%$, em relação ao período VB3. O resultado de 22,6\% em VB3 para o indicador NCG evidencia a tendência de destinação de recursos para outra forma de aplicação, podendo ser financeira ou permanente, no sentido de ajuste ao perfil da estrutura financeira desejada no período de crise.

A aplicação da modelagem Fleuriet permitiu visualizar a redução no número de empresas no Tipo 2 de situação financeira sólida, e aumento no Tipo 3 situação financeira insatisfatória, em períodos relacionados ao advento da crise no Brasil, tal fato corrobora, combinado com o entendimento dos demais resultados, na inferência de que a crise financeira de 2008 exerceu influência negativa em meio ao setor industrial brasileiro.

Por fim, ressalta-se que o aspecto financeiro anterior a crise para as indústrias brasileiras, na média, mostrava-se favorável, com forte tendência de crescimento em meio ao ambiente macroeconômico nacional, e também no mercado de exportação. Contudo, o advento da crise reteve o crescimento da economia e exigiu ajustes de estrutura financeira por parte das indústrias no sentido de se adequarem a nova realidade, assim como de se prepararem para a retomada do crescimento no período pós-crise. Pois, conforme o conjunto de dados evidenciados neste estudo se constata a redução na disponibilidade de recursos em meio ao período da crise, mas logo após, identificam-se esforços no sentido de retomar a produtividade e o crescimento, perceptível em meio às práticas de gestão do capital de giro evidenciadas nos demonstrativos contábeis. 


\begin{abstract}
This study's main objective is to identify the level of liquidity of companies in the Brazilian industrial sector by traditional analysis and dynamics of working capital, amid the financial crisis of 2008. The sample consists of 31 companies listed in the Exame Melhores e Maiores Magazine in 2009 with shares traded on the BM\&FBOVESPA (São Paulo Stock exchange). It was developed a descriptive analysis with qualitative and quantitative approach. Among the study's findings, stands out: spread measures monitored by the application of descriptive statistics in the traditional liquidity indicators indicate a tendency of reduction of the distance between the companies regarding the level of financial liquidity; despite positive indicators, there is variation gross negative in the period VB3, for indicators liquidity drought (LS), current liquidity (LC) and overall liquidity (LG) in the level of $77.4 \%, 74.2 \%$, and $61,3 \%$, respectively; the treasury balances (ST) indicator demonstrates limitation for an immediate recovery of levels of liquidity at the end of the period of the crisis, therefore the period of crisis surpassed two quarters for the Brazilian industrial sector. The modeling Fleuriet allowed view the reduction in the number of companies in Type 2 of situation financial solid, and an increase in Type 3 of situation unsatisfactory financial in periods related to the advent of the crisis in Brazil.
\end{abstract}

Key-words: financial crisis; brazilian industrial sector; working capital.

\title{
Referências
}

ASSAF NETO, A. Estrutura e análise de balanços: um enfoque econômico-financeiro. 9. ed. São Paulo: Atlas, 2010. .; SILVA, C.A.T. Administração do Capital de Giro. 3. ed. São Paulo: Atlas, 2010.

ÁZARA, L. N. de; PASSANHA, G. R. G.; ÁZARA, L. N. de. Os efeitos da crise de 2008 sobre a volatilidade dos retornos das ações bancárias no Brasil. In: SEMEAD, 13, 2010, Anais... São Paulo: SEMEAD, 2010. Disponível em: <http://www.ead.fea.usp.br/semead/13semead/resultado/trabalhosPDF/650.pdf>. Acesso em: 20 set. 2010.

BEZEMER, D. J. Understanding financial crisis through accounting models. Accounting, Organizations and Society, Groningen, aug. 2010. Disponível em: 〈www.elsevier.com/locate/aos〉. Acesso em: 29 out. 2010.

BIZOTTO, B. L. S.; COSTA, C. A.; RUI, C.; LAZZAROTTO, E.; RECHE, R. A.; CAMARGO, M. E. Estudo de caso dos impactos da crise global nos critérios competitivos estratégicos de empresas. Revista Gestão Industrial, Ponta Grossa, v. 7, n. 1, 2011.

\section{cross $r$ ref}

BRAGA, R. Análise avançada do capital de giro. Caderno de Estudos, São Paulo, FIPECAFI, n. 3, p. 1-20, set. 1991.

; NOSSA, V.; MARQUES, J. A. V. da C. Uma proposta para a análise integrada da liquidez e rentabilidade das empresas. Revista Contabilidade \& Finanças, São Paulo, ed. Esp., p. 51-64, jun. 2004.

BRASIL. Ministério da Fazenda. Receita Federal do Brasil - RFB. Dados gerais da balança comercial. Disponível em: <http://www.receita.fazenda.gov.br/Historico/Aduana/Balança/2008/dezembro/dadosgerais.htm>. Acesso em: 07 ago. 2010.

; Ministério do Desenvolvimento, Indústria e Comércio Exterior. Anuário estatístico 2011. Disponível em: < http://www.mdic.gov.br/sitio/interna/interna.php?area=2\&menu=1479>. Acesso em: 07 ago. 2011.

CAGLIOLO, M. O.; FERRARO, G.; METILLI, G. Realidad de las pymes Argentinas ¿remedios o terapias ante la crisis? un aporte desde la gestión de costos. In: XI CONGRESO INTERNACIONAL DE COSTOS Y GESTIÓN. 2009, Anais... Trelew: Instituto Internacional de Costos, 2009, CD-ROM.

CINTRA, M. A. M.; FARHI, M. A crise financeira e o global shadow banking system. Novos Estudos - CEBRAT, São Paulo, n. 82, nov. de 2008. 
COOPER, D. R.; SCHINDLER, P. S. Métodos de Pesquisa em Administração. 7. ed. Porto Alegre: Bookman, 2003.

EXAME. Melhores e maiores 2009. São Paulo: Ed. Abril, jul. 2009.

FLEURIET, M.; KEHDY, R.; BLANC, G. O modelo Fleuriet: a dinâmica financeira das empresas brasileiras. 7. ed. Rio de Janeiro: Elsevier, 2003.

MARQUES, J. A. V. da C.; BRAGA, R. A análise dinâmica do capital de giro: o modelo Fleuriet. Revista de Administração de Empresas. São Paulo: v. 35, n. 3, p. 49-63, mai./jun. 1995.

MARTINS, B. S. Financial instability and credit constraint: evidence from the cost of bank financing. Working Paper Series, Brasília, n. 221, nov. 2010.

MATIAS, A. B. (coord.) Finanças corporativas de curto prazo: a gestão do valor do capital de giro. São Paulo: Atlas, 2007.

MESQUITA, M.; TORÓS, M. Considerações sobre a atuação do Banco Central na crise de 2008. Trabalhos para discussão, Brasília, n. 202, mar. 2010.

RICHARDSON, R. J. Pesquisa social: métodos e técnicas. 3. ed. São Paulo: Atlas, 1999.

ROSS, S. A.; WESTERFIELD, R. W.; JAFFE, J. F. Administração financeira. 2. ed. São Paulo: Atlas, 2009.

SENA, B. H. S.; SANTANA, C. M.; RODRIGUES, R. J. D.; ARRIO, K.; MACHADO, C. A.; ROCHA, D. G. da. Uma análise dos fatos relevantes de companhias com registro na CVM utilizando aprendizagem de máquina: estudo após a crise financeira. In: ENANPAD, 33, 2009, Anais... São Paulo: ANPAD, 2009, CD-ROM.

SOUSA, A. F.; LUPORINI, C. E. M.; SOUZA, M. S. Gestão do capital de giro. Caderno de Pesquisas em Administração, São Paulo, v. 1, n. 3, 2. sem. 1996.

UNITED STATES OF AMERICA - USA. The financial crisis inquiry report: final report of the National Commission on the causes of the financial and economic crisis in the United States. Washington: Official Government Edition, jan. 2011.

VIEIRA, M. V. Administração estratégica do capital de giro. 2. ed. São Paulo: Atlas, 2008.

ZANI, T. B.; ZANINI, F. A. M.; ZANI, J. Governança Corporativa e Conflito de Agência: Estudo de caso sobre a utilização de derivativos cambiais por cinco grandes empresas brasileiras. In: ENANPAD, 34, 2010, Anais... Rio de Janeiro: ANPAD, 2010, CD-ROM.

\section{Dados dos autores:}

Nome completo: Cristiano do Nascimento

Filiação institucional: Universidade Federal do Paraná - UFPR

Departamento: Departamento de Ciências Contábeis

Função ou cargo ocupado: Mestrando em Contabilidade

Endereço completo para correspondência (bairro, cidade, estado, país e CEP): Rua Isaltina de Assunção Farias, 331, Bairro Rio Tavares - Florianópolis-SC - Brasil - CEP: 88048-416.

Telefones para contato: (48) 8806-4274

e-mail: cristiano.do.nascimento@live.com 
Nome completo: Márcia Maria dos Santos Bortolocci Espejo

Filiação institucional: Universidade Federal do Paraná - UFPR

Departamento: Departamento de Ciências Contábeis e Coordenadora do PPG Mestrado em Contabilidade.

Função ou cargo ocupado: Professora adjunta.

Endereço completo para correspondência (bairro, cidade, estado, país e CEP): Av. Prefeito Lothário Meissner, 632/1 andar, Jardim Botânico - Curitiba-PR - Brasil - CEP: 80210-170.

Telefones para contato: (41) 3360-4417

e-mail: marciabortolocci@ufpr.br

Nome completo: Simone Bernardes Voese

Filiação institucional: Universidade Federal do Paraná - UFPR

Departamento: Departamento de Ciências Contábeis e Coordenadora do PPG Mestrado em

Contabilidade

Função ou cargo ocupado: Professora Adjunta

Endereço completo para correspondência (bairro, cidade, estado, país e CEP): Av. Prefeito Lothário Meissner, 632/1 ${ }^{\circ}$ andar, Jardim Botânico - Curitiba-PR - Brasil - CEP: 80210-170.

Telefones para contato: (41) 3360-4417

e-mail: sbs1611@ hotmail.com

Nome completo: Elisete Dahmer Pfitscher

Filiação institucional: Universidade Federal de Santa Catarina - UFSC

Departamento: Coordenadora de Tutoria, Pesquisa e Monografias do Ensino a Distância.

Função ou cargo ocupado: Professora Adjunta

Endereço completo para correspondência (bairro, cidade, estado, país e CEP): Rua Mário Cândido da Silva, 46, Bairro Itaguaçu - Florianópolis-SC - Brasil - CEP 88085-475.

Telefones para contato: (48) 3721-9383

e-mail: elisete@cse.ufsc.br

Nome completo: João Teles

Filiação institucional: Universidade Federal de Santa Catarina - UFSC

Departamento: Departamento de Ciências Contábeis

Função ou cargo ocupado: Mestrando em Contabilidade

Endereço completo para correspondência (bairro, cidade, estado, país e CEP): Rua Professora Maria Julia Franco, 49, Bairro José Mendes - Florianópolis-SC - Brasil - CEP - 88020-280 
Telefones para contato: (48) 3223-8557

e-mail: joaoteles.contab@gmail.com

Enviado em: 08/08/2011

Aprovado em: 27/05/2013 